\title{
MECHANICAL PROPERTIES OF ORDERED MONODISPERSE POLYSTYRENE LATEX AS A LYOTROPIC LIQUID CRYSTAL
}

\author{
S. MITAKU, T. OHTSUKI and K. OKANO \\ Department of Applied Physics, Faculty of Engineering, University of Tokyo, Japan
}

\begin{abstract}
Résumé. - Le comportement du courant stationnaire et la viscoélasticité dynamique à $40 \mathrm{kHz}$ du polystyrène latex monodispersé et ordonné ont été mesurés. Les résultats indiquent que le latex ordonné est une sorte de cristal qui montre la rigidité de $10^{3}$ dyne $/ \mathrm{cm}^{2}$ environ, et la limite plastique très basse de 0,5 dyne $/ \mathrm{cm}^{2}$.

Abstract. - The steady flow behaviours and the dynamic viscoelasticity at $40 \mathrm{kHz}$ of the ordered monodisperse polystyrene latex have been measured. The results indicate that the ordered latex is a kind of crystal which shows the rigidity of about $10^{3}$ dyne $/ \mathrm{cm}^{2}$ and the very low yield stress of 0.5 dyne $/ \mathrm{cm}^{2}$.
\end{abstract}

1. Introduction. - An ordered crystalline array is formed in monodisperse polystyrene latex, i.e. an aqueous suspension of polystyrene spheres with uniform size, when the volume concentration of polystyrene spheres is sufficiently large [1]. Most of the properties associated with a crystalline solid have been found in the ordered structure of polystyrene spheres : the melting from ordered to disordered state takes place [2], and shear as well as longitudinal modulus is finite in the ordered state $[3,4]$. However, the ordered suspension flows, as if its apparent viscosity is as low as that of the disordered suspension. We may, therefore, say that the regular lattice structure of polystyrene spheres is a lyotropic liquid crystal that has long range translational order but flows like liquids.

Based upon a phase diagram of the ordering of polystyrene spheres obtained by Hachisu et al. [5], Wadati and Toda [6] have pointed out that the ordered structure is due to the electrostatic repulsive interaction between polystyrene spheres and analogous to the ordering phenomena of hard spheres predicted by computor simulations [7]. Several studies have been reported on the order-disorder transitions in monodisperse polystyrene latex [8-11], and the ordering of polystyrene spheres by purely repulsive interaction appears to be established as far as the phase diagram is concerned.

Previously, we have measured the viscoelasticity of the monodisperse polystyrene latex by a torsional quartz crystal methods and have shown that the ordered structure has the rigidity of about $10^{3}$ dyne $/ \mathrm{cm}^{2}$ [12]. Furthermore, the ionic concentration dependence of the rigidity has been explained theoretically based upon the electrostatic repulsion between polystyrene spheres [11].

In the present work, we have measured the flow behaviours of the ordered monodisperse latex by a concentric cylinder viscometer [13] together with the viscoelasticity at $40 \mathrm{kHz}$ and the yield stress of about $0.5 \mathrm{dyne} / \mathrm{cm}^{2}$ have been observed for the first time.

2. Methods and materials. - The relation between the shear stress and the shear rate was determined by using a concentric cylinder viscometer specially designed for the measurements in the shear rate regions between $5 \times 10^{-5}$ and $60 \mathrm{~s}^{-1}$ [13]. This apparatus is developed from the viscometer described by Zimm and Crothers [14] and the scanning of the shear stress is possible from $4 \times 10^{-3}$ to 2 dyne $/ \mathrm{cm}^{2}$.

The viscoelasticity at $40 \mathrm{kHz}$ is measured by a torsional quartz crystal method [12]. A composite torsional quartz transducer has been employed in order to apply this method to aqueous conductive liquids. The surface of the transducer is partly gilded to prevent both the wetting of the transducer surface by water and the electric image force upon the charged polystyrene spheres. Details of the improvements will be described elsewhere.

The diameter and the surface charge density of polystyrene spheres are important characteristics in analyzing the measurements of mechanical properties [11]. About two hundred polystyrene spheres were examined by an electron microscope in order to determine the mean value and the standard deviation of diameter. The distribution of diameter is shown in figure 1 , which confirms the nearly uniform size 


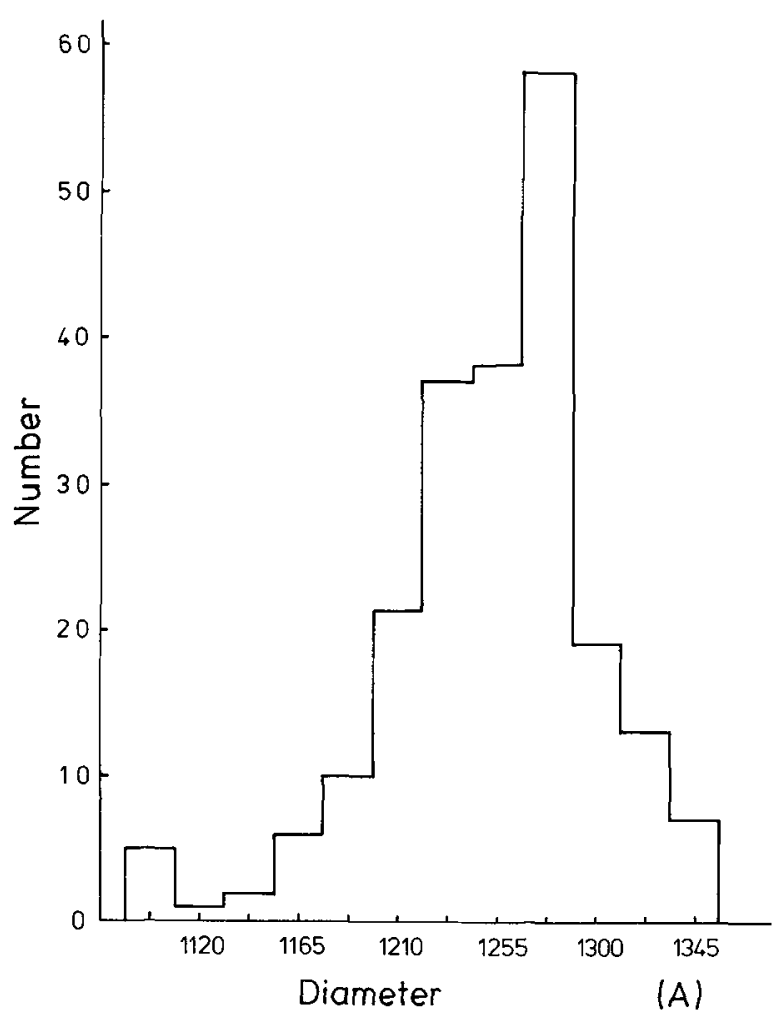

Fig. 1. - Particle size distribution of latex I.

of spheres. The mean diameter was $125 \mathrm{~nm}$ and its standard deviation was $5 \mathrm{~nm}$. The surface charge density was measured by a conductometric titration. Since the surface of polystyrene spheres is negatively charged by $\mathrm{SO}_{4}^{-}$radicals, the titration was performed by $\mathrm{NaOH}$. Figure 2 shows the results of the titration. There is a distinct minimum of the conductivity that corresponds to the neutralized point. The surface charge per a sphere was determined as 1240 electron charges from the amount of $\mathrm{NaOH}$ needed for the neutralization. This monodisperse latex (latex I) was used for the measurement of the ionic concentration dependence of the steady flow behaviours. The dynamic viscoelasticity was also measured in the latex I without added salt.

Suspension of polystyrene spheres (latex II) with the diameter of $118 \mathrm{~nm}$ and the charge density of 6800 was used for the measurement of the ionic concentration dependence of the viscoelasticity. The characteristics of these latexes are listed in table I.

\section{TABLE I}

\section{Characteristics of monodisperse latexes}

Diameter

Standard deviation

Charge per a particle

Volume concentration Lattice constant

$\begin{array}{cc}\text { Latex I } & \text { Latex II } \\ - & - \\ 125 \mathrm{~nm} & 118 \mathrm{~nm} \\ 5 \mathrm{~nm} & 5 \mathrm{~nm} \\ 1240 \text { electron } & 6800 \text { electron } \\ \text { charges } & \text { charges } \\ 5.85 \% & 15.3 \% \\ 291 \mathrm{~nm} & 200 \mathrm{~nm}\end{array}$

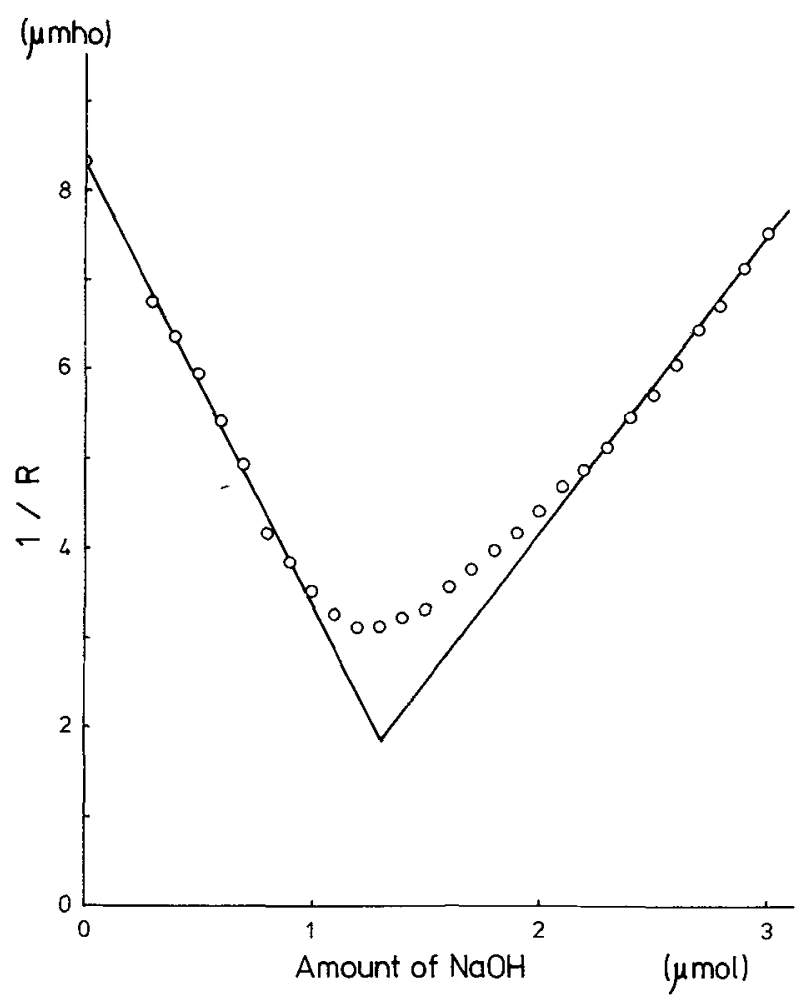

Fig. 2. - Conductometric titration of latex $\mathbf{I}$ by $\mathrm{NaOH}$.

Generally, the ordered structure is more stable when the volume concentration is higher, and the yield stress of the ordered structure of latex II was too high to measure the flow behaviours by our viscometer. Therefore, the measurement of the flow behaviours was carried out with the latex.I. On the other hand, it was difficult to measure systematically the ionic concentration dependence of the viscoelasticity in latex I, because the order-disorder transition occurred at very low ionic concentration.

3. Viscoelasticity. - The dynamic rigidity and viscosity of the latex II at $40 \mathrm{kHz}$ were measured by the torsional quartz crystal method in which a composite quartz transducer was employed. The results are shown in figure 3 . The rigidity was about $1.5 \times 10^{3} \mathrm{dyne} / \mathrm{cm}^{2}$ in the ionic concentration range below $100 \mu \mathrm{M}$ and decreased to $0.4 \times 10^{3} \mathrm{dyne} / \mathrm{cm}^{2}$ through the transition region from $100 \mu \mathrm{M}$ to $200 \mu \mathrm{M} \mathrm{KCl}$. This ionic concentration dependence of the rigidity in the latex is the same as the previously reported measurements at $70 \mathrm{kHz}$ [12], and the higher value of dynamic rigidity at low ionic concentrations is due to the ordered crystalline structure of polystyrene spheres. The iridescence that is caused by a Bragg reflection of visible light abruptly disappeared at the ionic concentration between $100 \mu \mathrm{M}$ and $200 \mu \mathrm{M}$. The dynamic viscosity, however, remained almost constant, $1.0 \sim 1.3 \mathrm{cp}$, and slightly decreased in the transition region.

According to the previously reported theoretical analysis of shear wave propagation in an ordered 


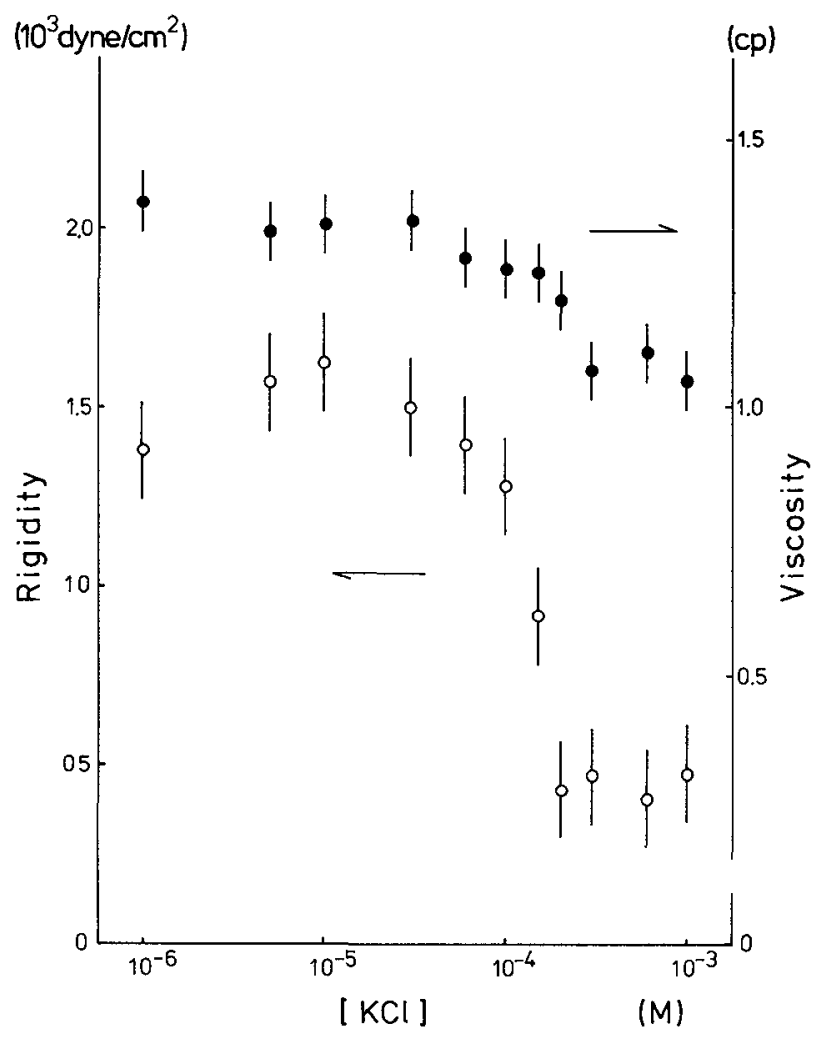

FIG. 3. - KCl concentration dependence of the rigidity $(O)$ and viscosity (๑) at $40 \mathrm{kHz}$ in the latex II.

suspension [11], the dynamic rigidity as obtained through the measurement of the mechanical impedance at the transducer surface is strongly depends on the coupling between the suspended particles and the transducer surface. When the coupling is strong enough as is the case where the surface is rather rough, in which the particles on the surface are forced to move without slip, the measured dynamic rigidity is equal to the static rigidity, while the dynamic viscosity does not show any marked change in the order-disorder transition. The present measurement of shear viscoelasticity is considered to correspond to this case of strong coupling. So we regard the measured dynamic rigidity as the static shear rigidity of the ordered suspension.

4. Steady flow properties. - We have also measured the flow behaviour of the monodisperse latex $I$. The shear rate is plotted in figure 4 as a function of the shear stress. When the ionic concentration is smaller than $32.5 \mu \mathrm{M}$, there exists an yield stress. The yield stress was 0.47 dyne $/ \mathrm{cm}^{2}$ and 0.05 dyne $/ \mathrm{cm}^{2}$ when the $\mathrm{KCl}$ concentration was 30 and $32.5 \mu \mathrm{M}$, respectively. The yield stress of the salt free latex was about $0.47 \mathrm{dyne} / \mathrm{cm}^{2}$. On the other hand, the latex suspension may be regarded as a Newtonian liquid when the ionic concentration is larger than $50 \mu \mathrm{M}$. The apparent viscosity decreases and approaches the value of the aqueous medium, when the ionic concentration is raised. Since the iridescence

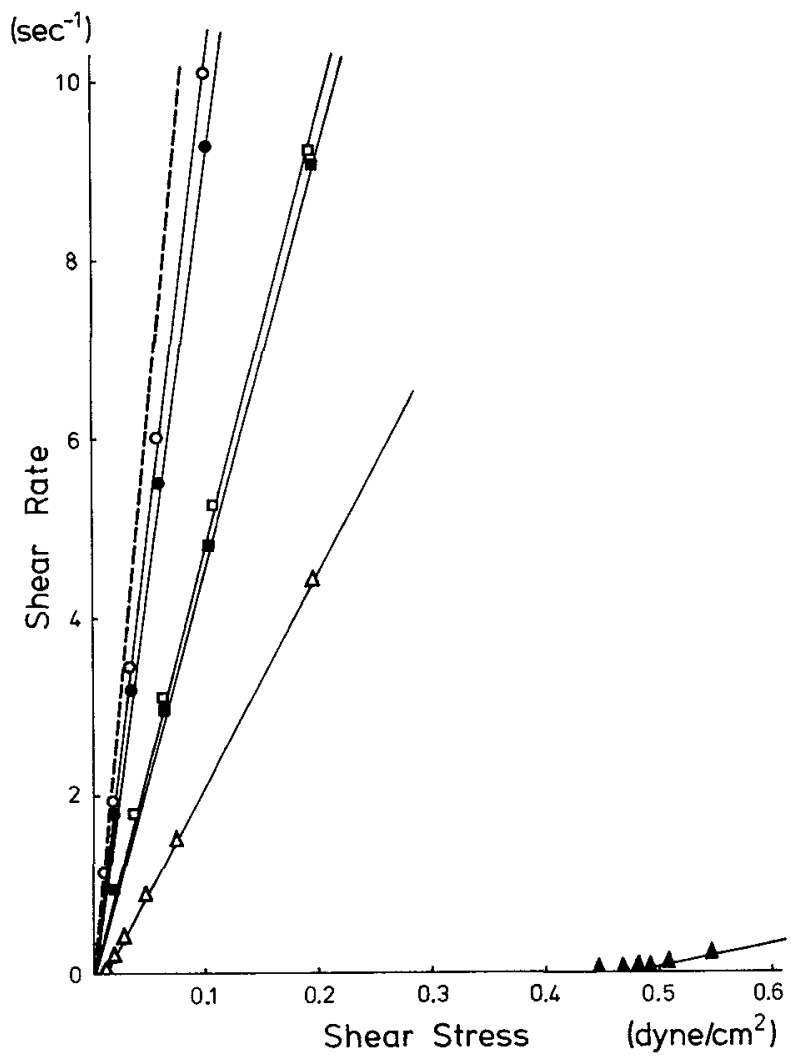

Fig. 4. - Flow curves of latex I that contains $\mathrm{KCl}$ of $30 \mu \mathrm{M}(\Delta)$, $32.5 \mu \mathrm{M}(\triangle), 50 \mu \mathrm{M}(\square), 100 \mu \mathrm{M}(\square), 300 \mu \mathrm{M}(\bullet)$ and $1 \mathrm{mM}(\mathrm{O})$. Broken line represents the flow curve of water.

of the ordered latex due to the Bragg reflection of visible light disappeared between 32.5 and $50 \mu \mathrm{M}$ of $\mathrm{KCl}$, this distinctive change in the flow behaviour has to be associated with the order-disorder transition of the polystyrene suspension.

The $\mathrm{KCl}$ concentration dependence of the yield stress and the specific viscosity for steady shear flow, $\eta_{\mathrm{sp}} \equiv\left(\eta-\eta_{0}\right) / \eta_{0}$, is shown in figure 5. At the transition point, the yield stress very sharply decreases from about $0.5 \mathrm{dyne} / \mathrm{cm}^{2}$ to zero, while the specific viscosity anomalously increases when the transition point is approached from the disordered region.

The existence of the yield stress is consistent with the finite rigidity we have measured and the yield stress shows similar dependence on $\mathrm{KCl}$ concentration to the rigidity. Since the measured rigidity of latex I under a salt free condition was

$$
5.5 \times 10^{2} \mathrm{dyne} / \mathrm{cm}^{2} \text {, }
$$

it appears that there is the following relation,

$$
\sigma_{0}=A G \text {, }
$$

where $\sigma_{0}$ and $G$ are the yield stress and the rigidity, respectively, and $A \simeq 8 \times 10^{-4}$. It is interesting to note that the equation (1) and the value of $A$ of about $10^{-3}$ have also been obtained in the ordinary crystalline solids like metals. As well known the very small value of $A$ in real crystals has been attributed to the 


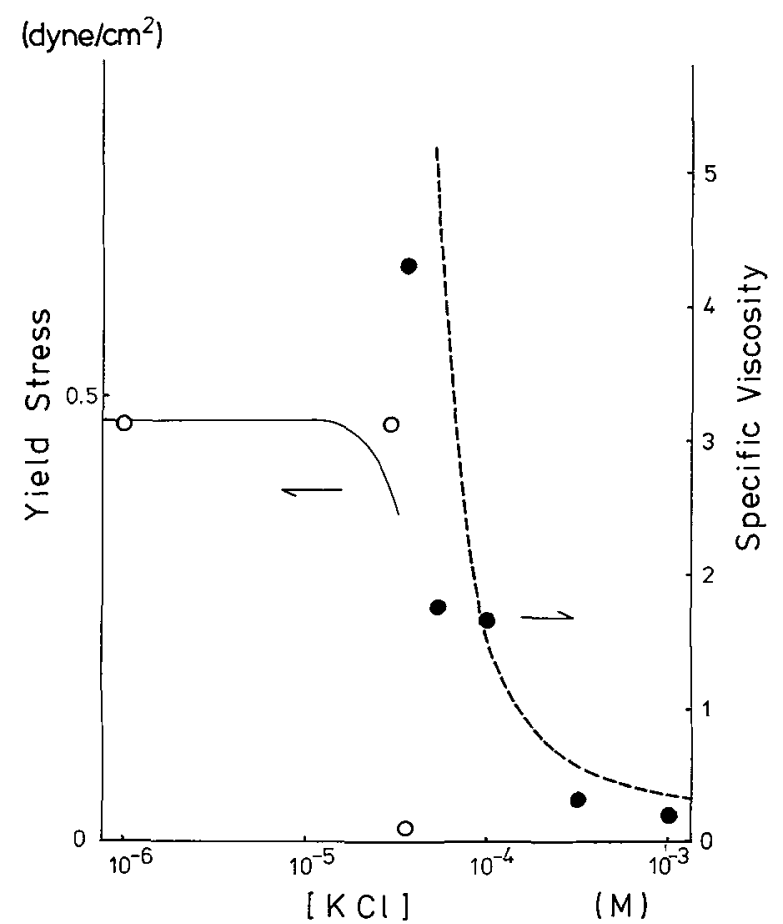

FIG. 5. - $\mathrm{KCl}$ concentration dependence of the yield stress $(0)$ and specific viscosity (e) of latex I.

existence of dislocations in crystallites. Nabarro has calculated the coefficient $A$ for a simple cubic crystal on the assumption that the plastic deformation of crystal results from the movement of dislocations [15],

$$
A=\frac{2}{1-v} \exp \left(\frac{-2 \pi}{1-v}\right)
$$

in which $v$ is a Poisson's ratio. For example, $A$ is equal to $3.6 \times 10^{-4}$ when $v=0.3$. The fact that $A$ is about $10^{-3}$ in the ordered suspension suggests the existence of dislocations in the lattice structure of polystyrene spheres.

The abrupt change of the specific viscosity in the disordered region is remarkable and is different from the behaviour of dynamic viscosity shown in figure 3 . The steady flow viscosity steeply increases when the ionic concentration approaches the transition point. This anomalous phenomenon is approximately explained by assuming that the effective hydrodynamic radius of a polystyrene sphere is given by the sum of the actual radius and the Debye screening length. The broken line in figure 5 is obtained by substituting this effective volume fraction into Brinkman's formula of suspension viscosity,

$$
\eta_{\mathrm{rel}}=(1-\Phi)^{-5 / 2} \text {. }
$$

Fairly good agreement is obtained. When this effective volume fraction becomes sufficiently large, crystalline structure is formed, and the rigidity and the yield stress become finite.

5. Discussion and conclusion. - The lattice structure in the monodisperse suspension of polystyrene spheres is a kind of crystal that has the rigidity of about $10^{3}$ dyne $/ \mathrm{cm}^{2}$ and the well defined yield stress of about 0.5 dyne $/ \mathrm{cm}^{2}$. Its high fluidity in usual experiments is due to the fact that the value of the yield stress is very small. These features of ordered polystyrene suspensions seems to quite resemble to some lyotropic liquid crystals having long range translational order, in which, e.g., spherical particles or aggregates of amphiphiles form a regular lattice structure. These lyotropic liquid crystals may possess small but finite shear rigidities and they may also have small yield stresses for a steady shear flow measurement. We may also conjecture that the main cause of the long-range translational order of lyotropic liquid crystals is the repulsive part of the interaction between particles or aggregates.

Acknowledgments. - We would like to thank Professor Y. Wada for his valuable advice. We are also grateful to Dr. H. Fujita for his discussion and kind offer of monodisperse latexes. This work was partly supported by the grant in aid for scientific research, 255003.

\section{References}

[1] LuCK, V. W., KLIER, M. and WesSLAU, H., Naturwissenschaften 14 (1963) 485

[2] Schaefer, D. W. and Ackerson, B. J., Phys. Rev. Lett. 35 (1975) 1448 .

[3] Mitaku, S., Ohtsuki, T. and Oxano, K., Internal Friction and Ultrasonic Attenuation in Solids (University of Tokyo Press, Tokyo) 1977, p. 291.

[4] Crandall, R. and Williams, R., Science 198 (1977) 293.

[5] Hachisu, S., Kobayashi, Y. and Kose, A., J. Colloid Interface Sci. 42 (1973) 342

[6] Wadati, M. and Toda, M., J. Phys. Soc. Japan 32 (1972) 1147.

[7] WadATI, M. and Toda, M., Oyo Butsuri 42 (1973) 1160 [in Japanese].

[8] Brenner, S. L., J. Phys. Chem. 80 (1976) 1437.
[9] Fujixa, H. and Ametani, K., Japan. J. Appl. Phys. 16 (1977) 1091.

[10] Megen, W. and Snook, I., J. Colloid Interface Sci. 57 (1976) 40.

[11] Ohtsukr, T., Mitaku, S. and Okano, K., Japan. J. Appl. Phys. 17 (1978) 627.

[12] Mitaku, S., Ohtsuki, T., Enari, K., Kishimoto, A. and OKano, K., Japan. J. Appl. Phys. 17 (1978) 305.

[13] Mitaku, S., Ohtsuki, T., Hirakawa, K., Handa, H. and OKANO, K., J. Fac. Engineering, the University of Tokyo, 1978 (in press).

[14] Zimm, B. H. and Crothers, D. M., Proc. Nat. Acad. Sci. 48 (1962) 905.

[15] Nabarro, F. R. N., Proc. Phys. Soc. 59 (1947) 256. 